\title{
The Application of a Hypothesis-driven Strategy to the Sensitive Detection and Location of Acetylated Lysine Residues
}

\author{
John R. Griffiths, ${ }^{\text {a }}$ Richard D. Unwin, ${ }^{\text {a Caroline A. Evans, }}$, \\ Siân H. Leech, ${ }^{\mathrm{b}, *}$ Bernard M. Corfe, ${ }^{\mathrm{b}}$ and Anthony D. Whetton ${ }^{\mathrm{a}}$ \\ a Stem Cell and Leukaemia Proteomics Laboratory, Division of Cancer Studies, University of Manchester, \\ Manchester, United Kingdom \\ ${ }^{\mathrm{b}}$ Human Nutrition Unit, Medical School, University of Sheffield, Royal Hallamshire Hospital, Sheffield, \\ United Kingdom
}

\begin{abstract}
The@application@of@a@hypothesis-driven@method@for@the@sensitive@determination@of@ysine acetylation@ites@n@nzymatically@igested@roteins@s बescribed.@omparative@ensitivity@ests were@arried@ut@sing@erial@ilution@f@n@cetylated@ovine@erum@lbumin@AcBSA)@igest

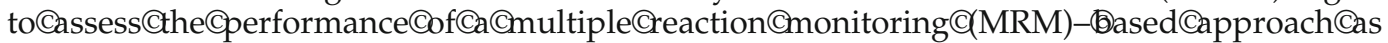
compared@o@@ore@onventional@recursor@canning(®P)@ethod.\$oth@ethods@ere@apable of@electively@etecting@n@cetylatedpeptide@t@he@ow@emtomoleđevel@hen@piked@nto@ background@f@00@mol@ix-protein@ryptic@igest.CThe@MRM@proach@was@oughly@enfold more@ensitive@han@recursor@canning@ith@ne@cetylated@eptide@etected@nd@equenced at@he@evel@f(2@mol@n-column.CTheథechnique@vas@ubsequently@pplied@o@@el-derived sample@f@ytokeratin-8@CK8)@hown@o@ontain@acetylated@ysine@esidues@by@Western@blot analysis.@The@strategy@applied@Cherein, Ctermed@MRM-initiated@detection@and@sequencing (MIDAS), $\odot$ resulted $\odot$ in $\odot$ the $\odot$ facile $\odot$ identification $\odot$ of $\odot$ novel $\odot$ sites $\odot$ of $\odot$ acetylation $\odot$ on $\odot$ this

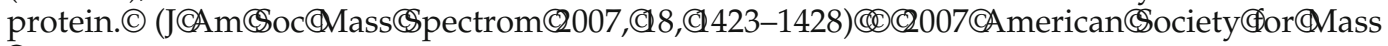
Spectrometry
\end{abstract}

\section{A} cetylation@is@an@important@posttranslational modification (PTM)@nvolved@n@he@egulation of@@umber@f(Rey@ellular@rocesses@including transcription $₫ 1]$, @protein-protein@interactions@2], @and protein@tabilityథ3].đhe@mportance@f@his@iologically relevant@modification@has@recently@been@compared@o that@f@hosphorylation $\$ 4$ ],@hich@s@@ey@egulator@f biological@rocesses, @and@hus@here@s@@rowing@eed for@the@sensitive@detection@and@location@of@sites@of acetylation@n甲roteins. $(9$ umber@f@nass@pectrometry (MS)-Based@nethods@have@been@ised@or@he@etermination@f(protein@cetylation \$5,৫].@ne@uch@pproach relies@n@he@etection@f@@haracteristic@eporter@on@t 126.1@h,@orresponding@o@he@mmonium@on@f@cetyl lysine@- $\mathrm{NH}_{3}$,@pon@ollisionally@activated@issociation (CAD)\$6].Althoughథhis@s@indeed@@ensitive@pproach, andథhas@he@dded@dvantage@f(being@ompatible@ith additional $\subseteq$ protein/peptide $($ modifications, $(\subset)$ a $@$ more promising@trategy@ith@egard@o@ensitivity@ould@e to@se@@ultiple@eaction (nonitoring $(\mathrm{MRM}) \oplus$ ransition as@he@riggerథor@n@information-dependent@cquisition

Address reprint requests to Prof. Anthony D. Whetton, University of Manchester, Division of Cancer Studies, Kinnard House, Kinnard Road, Manchester M20 4QL, UK. E-mail: Tony.Whetton@manchester.ac.uk

* Current address: Department of Oral Pathology, School of Clinical Dentistry, University of Sheffield, Sheffield, S10 2TA, UK
(IDA)@xperiment@ind@enerate@equence@information from@@ubsequent@product@on@san.CThis@echnique, termed MIDAS-for MRM-initiated detection and sequencing - has been previously applied to phosphorylation studies and was shown to be on the order of ten times more sensitive than the commonly used -79

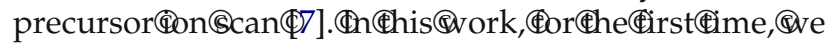
demonstrate a similar improvement in sensitivity of a MIDAS-based acetylation method over an existing precursor scanning approach.

\section{Experimental}

\section{Sensitivity Comparison}

Acetylated bovine serum albumin (AcBSA, Invitrogen, Paisley, UK) was diluted to 15 pmol $\mu \mathrm{L}^{-1}$ in $100 \mu \mathrm{L}$ of $25 \mathrm{mM}$ ammonium bicarbonate and digested at $37^{\circ} \mathrm{C}$ overnight by addition of modified porcine trypsin (Promega, Southampton, UK) at a 50:1 total protein:enzyme ratio. The digest was dried and resuspended in $150 \mu \mathrm{L}$ of $2 \%$ (vol/vol) acetonitrile $/ 0.1 \%$ (vol/vol) formic acid, providing a stock concentration of $10 \mathrm{pmol} \mu \mathrm{L}^{-1}$. The stock sample was further diluted in the same solvent containing a six-protein mix tryptic digest, to generate standard solutions. A single preparation of this AcBSA 
digest@as@sed@n@ll@xperiments.(The@ix-protein@ix sample,@consisting@of@trypsin-digested@BSA, Calcohol dehydrogenase,(Capo-transferrin, $(\mathcal{O} \beta$-galactosidase, $(\mathrm{Cly}$ sozyme, Cand@ytochrome-C, $@$ was @diluted@from@a@i 00 pmol@lyophilized@sample@ $($ LCCPackings, $C A m s t e r d a m$, The@Netherlands)@sing(\% $\%$ vol/vol)@cetonitrile/0.1\% (vol/vol)@ormic@cid@and@onstituted@he@background matrix@500@mol@n-column(loading).

\section{Preparation of CK8 Sample}

HCT116@olon@arcinoma@ells@ere@ultured@(\$) ulbecco's@modified@Eagle@medium $@$ Invitrogen), $@ 10 \% @(v o l /$ vol)@etal@alf@erum $₫$ Harlan@era@abs, $₫$ oughborough, UK), Cand@penicillin/streptomycin@(Invitrogen).@Cells were@reated@with@odium@utyrate@Calbiochem, $₫$ Nottingham,(UK)@s@ndicated.

Cytoskeletal@isolations@were@performed@mainly@as

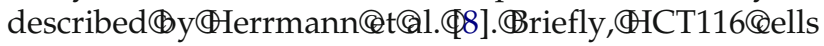
were@ultured@with@odium@utyrate $₫ 5 @ n M @$ Mor@7@). Low-detergent@ouffer $₫$ low $@$ buffer)@and (high-detergent buffer@high@uffer)@ere@prepared@s@etailed@reviouslyథ8].@థellet@ontaining@ytoskeleton@as@sed@or further@nalysis.

Samples@were@prepared@or@wo-dimensional@2-D) gels@using@the@Ettan®2-D@Clean-up@kit,@followed@by protein@ssay@using@he@ttan ${ }^{\mathrm{TM}}(2-D @$ uant $(k i t @$ Amer-

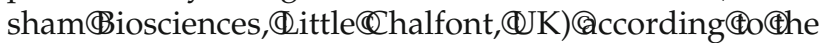
manufacturer's@instructions. $\mathbb{P}$ rotein@amples@were@e-

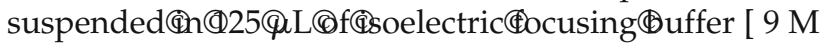

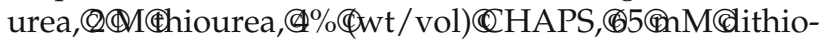

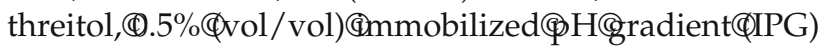
buffer@(Amersham@Biosciences, CLittle@Chalfont, (CUK)] and@oaded@nto®-cm@mmobilized@H@radient@trips

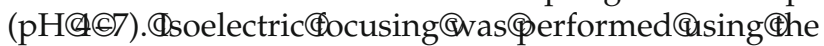
IPGphor ${ }^{\mathrm{TM}}(A$ Amersham@Biosciences)@t@20@C. CThe@econd@imension@was@a@standard@SDS-PAGE@protocol using $₫ 0 \% @$ niniథormat@els.@trips@ere@quilibrated $\oplus$ or

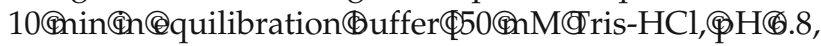

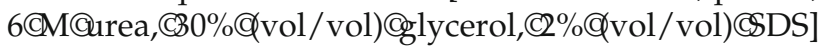

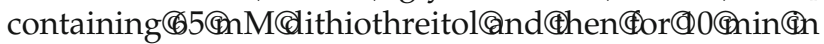
the@same@buffer@containing $₫ 240 @ \mathrm{mM}$ Miodoacetamide before@running(the@second@dimension. $\odot$ Gels@ $\odot$ were stained@sing@oomassie@lue@Sigma,(Poole,@U).@ngel@tryptic@digestions@were@performed@as@described previously $\Phi 9$ ].

\section{Liquid Chromatography and Mass Spectrometry}

For@each@experiment,®5@ $\mu$ L@of@digested@sample@was loaded@onto@a@15@cm@x @75@um đinner@iameter@(ID)

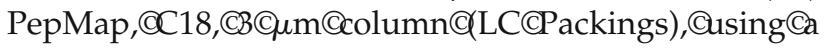
standard@LCCPackings@UltiMate@pump@and@FAMOS autosampler.đHPLC@uffers@A@and@B@onsisted@f(2\%

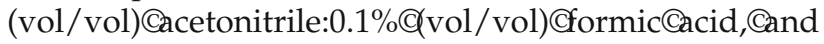

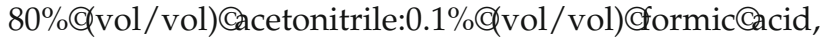
respectively.(\$amples@ere@esalted@n-line@efore@eparation@sing@@icroprecolumn $\$ 5 @ n m \propto \propto(300 @ u m(D)$ cartridge.CThe@washing@olvent@was@.1\%@ormic@id

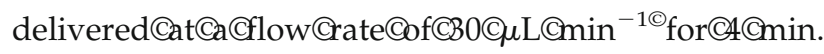
Peptides@ere@eparated@ver@Qgradient $₫$ rom $\$ \%$ (Bffer

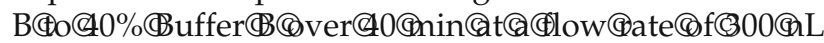
$\min ^{-1}$.@ Chromatography@was@performed@on-line@to@a 4000@TRAP@nass@pectrometer@Applied@iosystems, Framingham, @AA,@SA).

The@instrument@ettings@n@he@mass@pectrometer

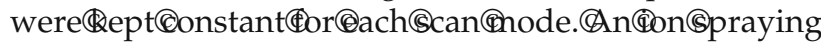
voltage@f@ 2300@@vas@sed@ith@@ource@as@etting

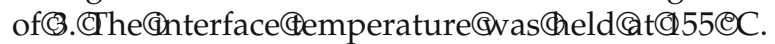

\section{MRM-initiated Detection and Sequencing (MIDAS)}

The@MIDASథrotocol,@hich@akes@ull@dvantage@f $₫$ he combined@unctionality@f(he@000@TRAP, (s) बescribed in@detail@elsewhere@7].@Briefly, Ca@triple@quadrupole MRM@can@s@sed@o@ensitively@elect@or@ostulated acetylated@eptides.@Under@ow-energy@ollision@onditions, @acetyl@ysine@esidues@have@een@hown@o@ragment,@producing@@iagnostic@on@t@m/z 126.1@orresponding@o@he@mmonium- $\mathrm{NH}_{3}$ ion@6].CA@able@f potential@acetylated@peptides@for@the@protein@under study@s@irst@enerated@ising@@oftware@cript@eveloped(†y@Applied@iosystems.@his@epresents@he@et@f precursor@nasses@o@e@equentially@ransmitted held@t@ow@esolution,@rhile@3@ras@eld@tatic@t@26.1 $\mathrm{m} / z$ (unit@esolution).(Limited@tudies@n@ur@aboratory suggest $₫$ hat@ptimal@ollision@nergies@o@acilitate@hese transitions@approximate@to@the@precursor@m/z $\times \mathbb{\complement} 0.1$ irrespective@f@harge@tate.@ollision@nergies@etween $50 \mathrm{Cand} \subset 80 \mathrm{CeV} @($ laboratory@frame@ofCreference)(were assignedథo@ach@ransition@epending@n@heథrecursor m/z such $₫$ hat@n/z of@bout $400 @ 60, @ E @-650 @$; $@$ bout $600-700, \mathrm{CE}=60 \mathrm{eV}$; about $700-800, \mathrm{CE}=70 \mathrm{eV} ;>800$, $\mathrm{CE}=80 \mathrm{eV}$.

Each step in the MRM list has an associated dwell time. Should a signal exceed a user-defined threshold (200 cps) following this period, then once all MRMs have been completed the instrument is instructed to switch modes (enhanced product ion, with Q3 now functioning as a linear ion trap) to perform three MS/MS scans before once again repeating the full list of MRM transitions. The total cycle time for the sensitivity comparison, including MS/MS switching, was $7.7 \mathrm{~s}$, which is equivalent to a 40-ms dwell time for each of the 72 MRM transitions.

\section{Precursor Scanning at $\mathrm{m} / \mathrm{z} 126.1$}

Precursor scanning is a selective and sensitive scanning technique used on triple quadrupole mass spectrome-

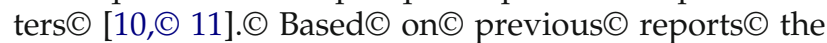
immonium $-\mathrm{NH}_{3}$ ion of acetyl lysine $(\mathrm{m} / \mathrm{z}$ 126.1) is a more sensitive and selective reporter ion for precursor

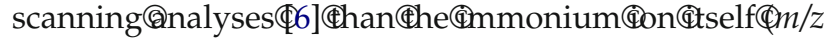
143.1)థ12].@3@ras@herefore@eld@tatic@t@26.1@h@ith resolution set to unit, whereas Q1 was scanned over the 

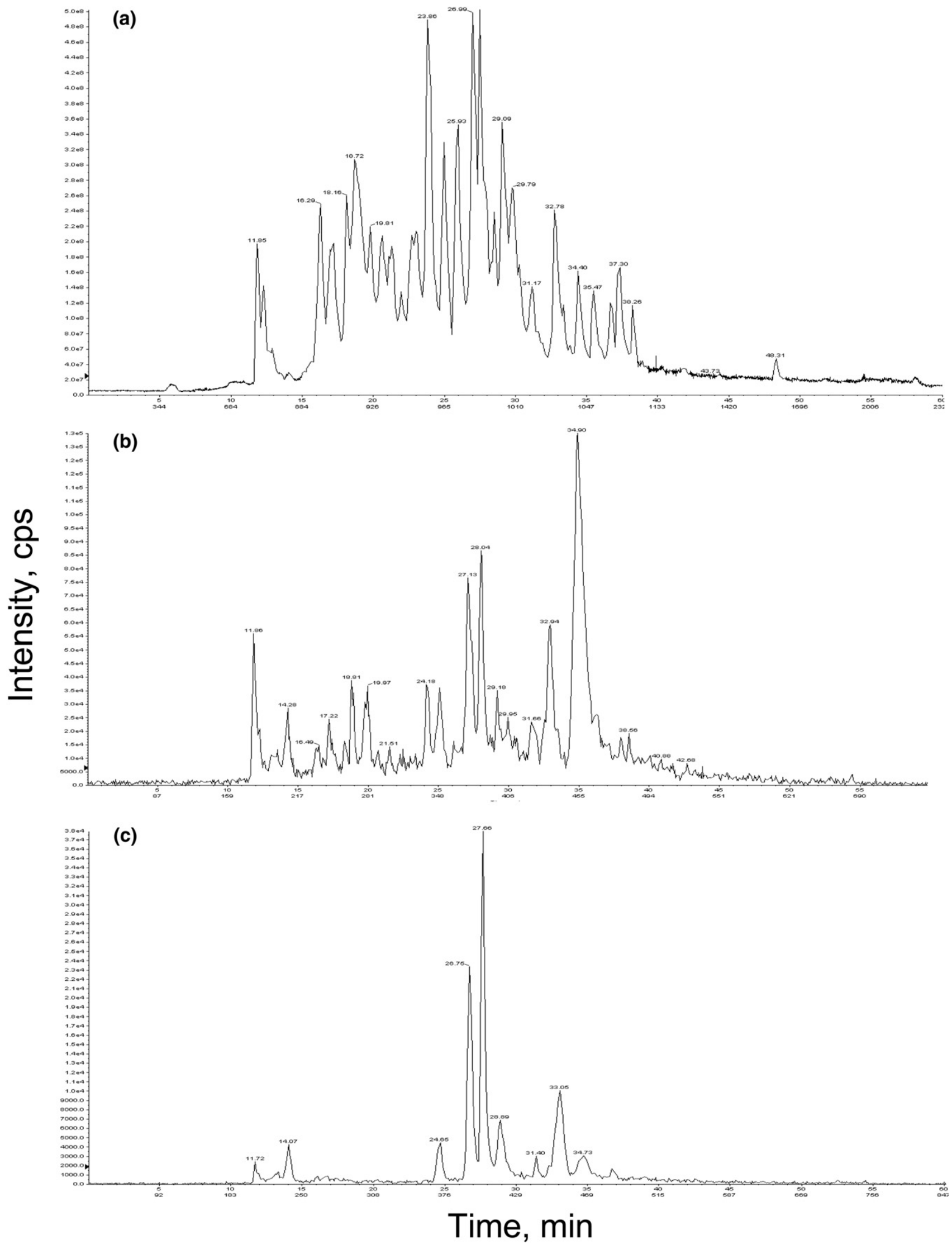

Figure 1. Total ion chromatogram (TIC) of $10 \mathrm{fmol}$ acetylated BSA in $500 \mathrm{fmol}$ six-protein mix using (a) information-dependent acquisition (TIC of EMS), (b) precursor ion scanning at $\mathrm{m} / \mathrm{z}=126.1$ (TIC of precursors), and (c) MIDAS (TIC of MRM) analysis. 


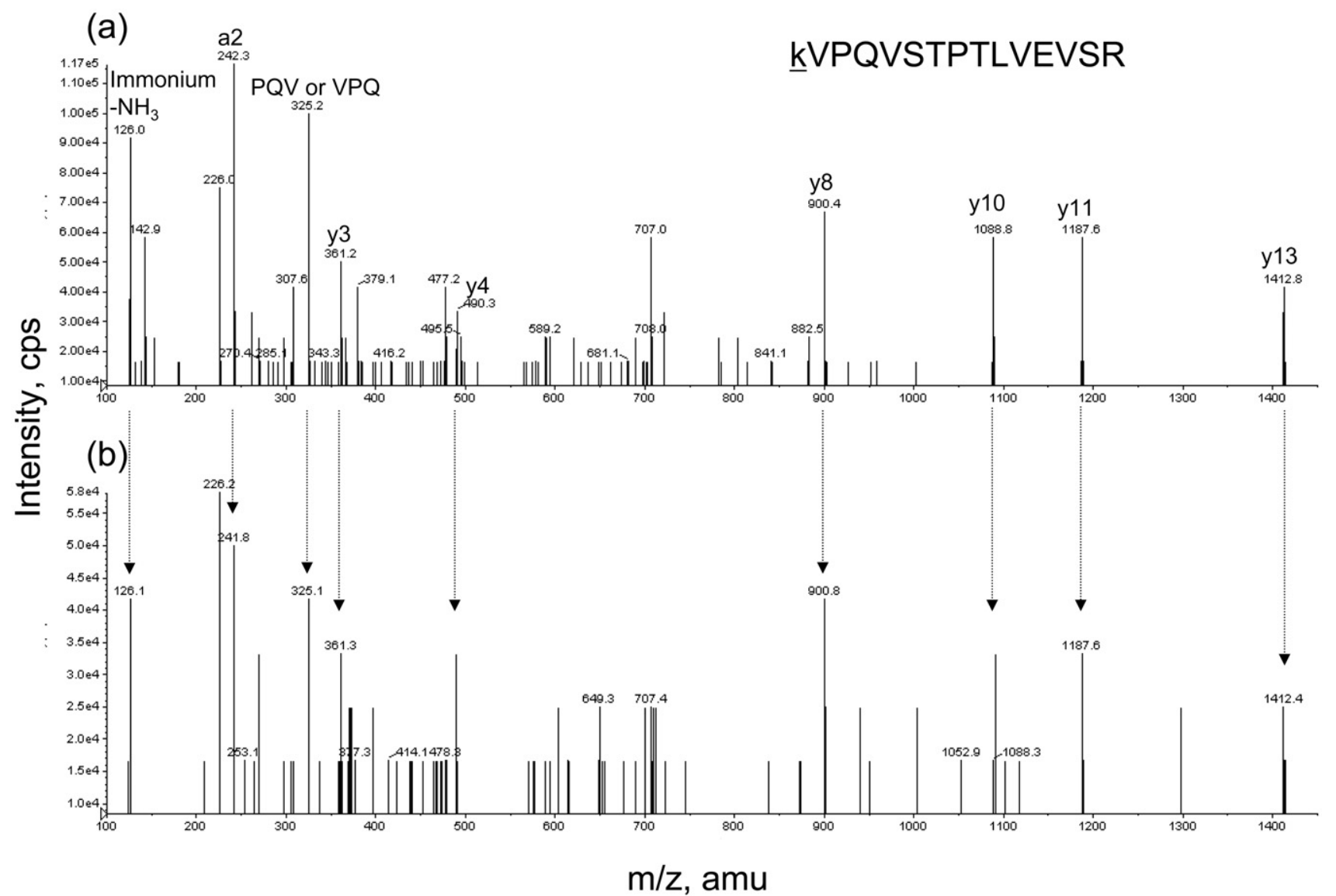

Figure 2. MS/MS spectra for the acetylated BSA peptide $k$ VPQVSTPTLVEVSR upon MIDAS analysis of (a) $5 \mathrm{fmol}$ and (b) $2 \mathrm{fmol}$ acetylated BSA in $500 \mathrm{fmol}$ six-protein digest on-column loading. $k$ denotes an acetylated lysine residue.

mass@ange@50@1200@Th@ver@@eriod@f 3 s with a 1 amu@tep@ize@resolution@et@o@ow).đThe@ollision@ell was@amped@ver(50@80@V@laboratoryథrame@f@eference)@uring@ach@can.CAnđDA@rigger@hreshold@f 1000@ps@vas@et, @and@ubsequent@MS/MS@ata@vere acquired@from@100@to@1500@Th.CTheCtotal@cycle@time including@MS/MS@witching@vas@.2@.

\section{Results and Discussion}

\section{Sensitivity Evaluation}

Acetylated BSA (AcBSA) was used to assess the sensitivity and suitability of the MIDAS method compared to precursor scanning for this application. A dilution series of trypsin-digested AcBSA in the range 50 to 1 fmol (on-column loading) was prepared, spiked into a $500 \mathrm{fmol}$ six-protein digest and analyzed in triplicate.

First, an MS-driven IDA experiment was carried out with $10 \mathrm{fmol}$ AcBSA in a $500 \mathrm{fmol}$ six-protein digest injected on-column. The background proteins were easily identified, although the level of acetylation was below the threshold detectable for all peptides using this approach (data not shown). A more focused strategy was therefore required to identify lower levels of acetylation. Both MIDAS and the precursor scanning methods offer an improvement in sensitivity and selectivity.๑igure@@hows@heథotal@on@hromatogram $₫$ TIC) for@MS-driven@DA@Figure@a), \$recursor@canning@t

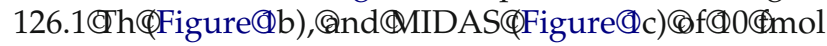
AcBSA in a 500 fmol six-protein digest. This figure demonstrates how the MS-driven IDA experiment spends the majority of the duty cycle switching to MS/MS mode on prominent nonacetylated peptides at the expense of the less abundant acetylated peptides. In contrast, both precursor ion scanning and MIDAS are tuned to select for only those peptides that are likely to be acetylated (i.e., release an ion of $\mathrm{m} / \mathrm{z} 126.1$ upon fragmentation) and therefore suffer from much less interference in the respective TIC. This figure also demonstrates how relatively uncluttered the MIDAS scan@is@(Figure@1c)@compared@to@the@precursor@scan (Figure@b).

To ensure a valid comparison between the two methods, it was necessary to keep both cycle times approximately the same. Thus, a cycle time, including any MS/MS scans, was set to about $8 \mathrm{~s}$, with the initial triggering scan (MRM or precursor scan) having a cycle time of about $3 \mathrm{~s}$. The consequence of this was a dwell 


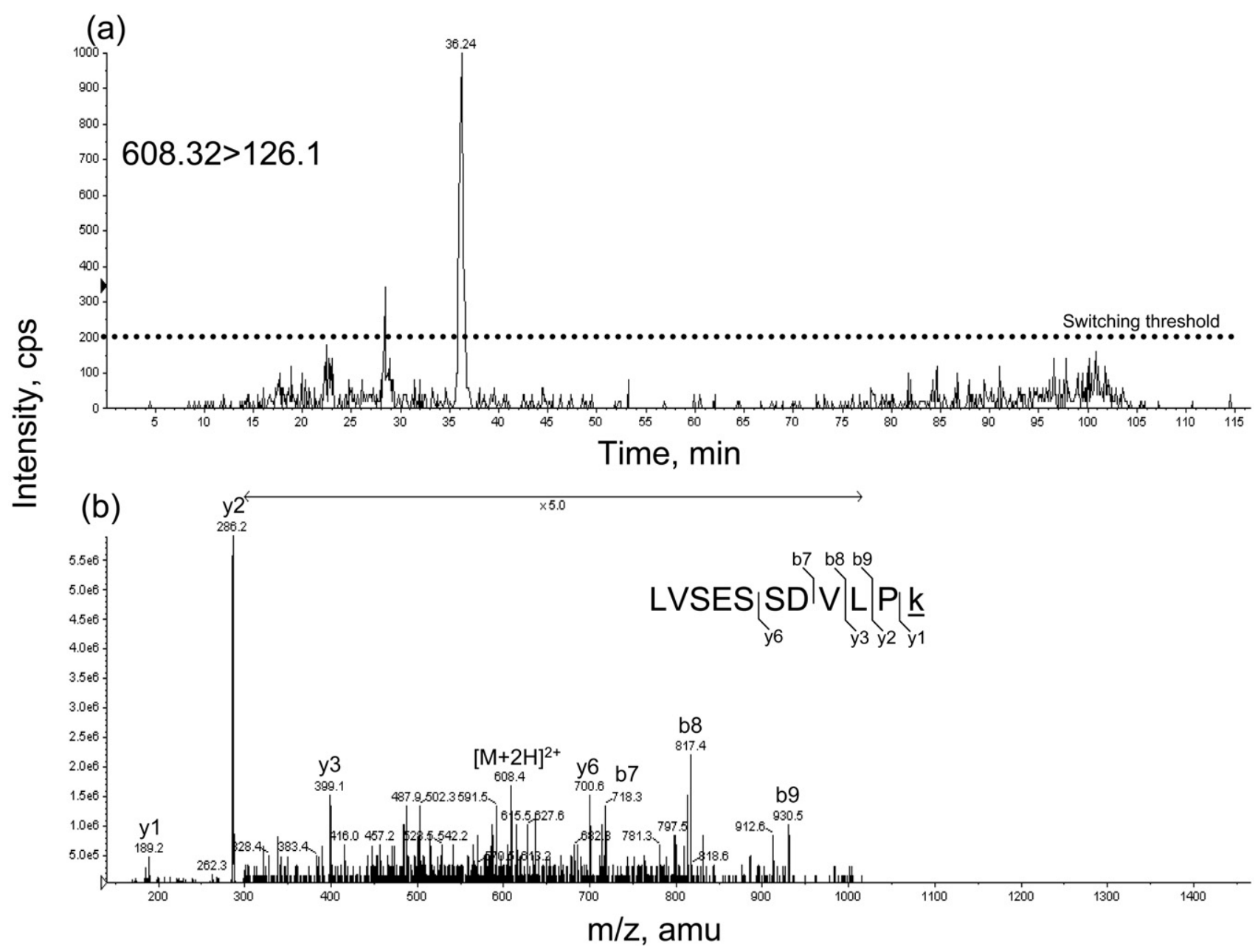

Figure 3. MIDAS experiment for the acetylation of CK8 showing (a) extracted ion chromatogram (XIC) for the MRM transition $608.32 \rightarrow 126.1$ with a switching threshold of 200 counts per second (cps), and (b) the annotated MS/MS spectrum generated for the acetylated peptide LVSESSDVLPk, where $k$ denotes an acetylated lysine residue.

time@f@0@ms@or@ach@MRM@ransition@72@ransitions with@5-ms@ause@between@ach)@nd@@orresponding "dwell@ime"థfor@heథS@f@4@nsథ450@1200@mu@n@3@ with@1@amu@step@size).CFrom@this@alone,Cone@would

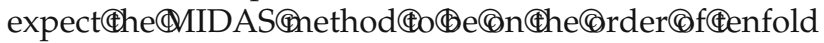
greater@ensitivity@han@heథS@method@or@his@rotein. This@vas@ndeed@ound@o@e@he@ase.@igure@2@hows

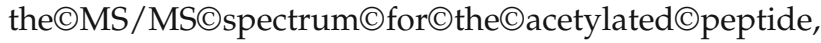
kVPQVSTPTLVEVSR $@$ (where $\mathbb{C}$ denotesCan@acetylated lysine@esidue), (atected@t(5)mol@AcBSA@n@(500@mol six-protein@igest $\Phi$ Figure@a)@ndQ(4) $@$ AcBSA $\Phi$ Figure 2b)@sing@MIDAS.đhis@eptide@vas@learly@etected@n

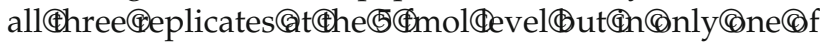
the@2@mol@amples, @ndicating@hat@ange@or@eliable level@f@etection.@n@ontrast,@50@mol@fCAcBSA@vas required@or@imilar@dentification@ising@he@recursor scan@pproach@data@iot@hown).

It@is@also@worth@noting@that@the@MIDAS@method detected@n@icetylated@eptide@ALkAWSVAR)Gn@he six-protein digest when no AcBSA was added. Using precursor scanning this peptide was detected with only $50 \mathrm{fmol}$ AcBSA present.

\section{Analysis of Cytokeratin 8}

Having confirmed that MIDAS was superior to both precursor scanning and IDA analysis on our instrument for identification of acetylation sites on a simple peptide mixture, we next demonstrated the application of this technique to a biological system by probing lysine acetylation on the intermediate filament protein, cytokeratin 8 . Intermediate filaments (IFs) are cytoskeletal structures that are crucial for maintaining the structural and mechanical integrity of@ells@nd@issuesథ13].@ytokeratin@@ @as@artially purified from cells by enrichment of the cytoskeletal fractions@using@differential@detergent@solubility@8]. Proteins were resolved using gel electrophoresis. Western immunoblot analysis with anti cytokeratin 8 and anti-acetyl lysine antibodies detected the presence of cytokeratin 8 and an acetylated protein of similar molecular weight in the cytoskeletal-enriched fractions (data not shown). The acetylation status of cytokeratin 8 was analyzed by mass spectrometry using the MIDAS approach based on detection of the 
126.1@n/z diagnostic@onథ(Đecause@his@as@etermined to@e@he@nost@ensitive@pproach.

A 5- $\mu$ L@liquot@f@he@ytokeratin@\&@igest@econstituted@n@uffer@@as@injected@n@olumn@nd@nalyzed using@@MIDAS@rotocol.@hree@MRM@ransitions@elatingథoథreviously@etermined@onacetylatedథeptides@f cytokeratin@ @SELEAALQR,CASLEAAIADAEQR,@nd

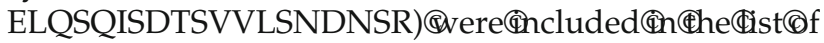
84@MRM@riggers@o@onfirm@he@rotein@dentity@nd@o check@uccessful@nzymatic@igestion.@hese@ransitions had@@horter@well@ime@20@ms)@han@he@redicted

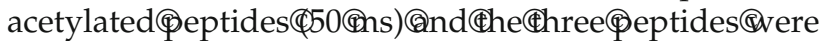
identified@by@theCanalysis@confirming@theđidentity@of CK8.CInCaddition, Cfive@novel@acetylation@sites@were identifiedథunpublished@bservations).đypical@ata@or the@acetylated@peptide@LVSESSDVLPk (AcK482)Care

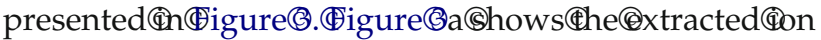
chromatogram@for@theCtransition@608.32@ $\rightarrow$ C126.1.(A) prominent@eak@t@@etention@ime@f(36.24@nin@ith@ signal@f@bout@000@ps@s@hown.®ecause@his@ignal breached@heథreset@witching@hreshold@f(200@ps, $₫$ he instrument@witched@nodes@nd@cquired@MS/MS@ata enabling@unambiguous@onfirmation@f@he@cetylation (Figure@3b).

\section{Conclusion}

The $($ application $(\subset$ of $($ a $(\subset$ hypothesis-driven $($ analysis (MIDAS)@for@the@determination@of@acetylated@ysine residues@in@a@mixture@of@tryptic@peptides@has@been demonstrated@for@the@first@time.CInitial@experiments using@cetylated@SA@s@@nodel@ompound@how@n approximately@0-fold@mprovement@n@ensitivity@ver theđ26.1@m/z precursor@canning@pproach@n@@000 QTrap.@hen@pplied@o@he@nalysis@f@@et@f@oomassie@lue-stained@2-D@el@pots,@ontaining@ytokeratin@8,Cfive@peptides@incorporating@acetylated@ysine residues@ere@etected@nd@equenced,థeading@o@heir unambiguous@ssignment.CThis@pplication@ote@emonstrates@hat@he@MIDAS@pproach@nay@e@pplied@o posttranslational@modifications@ther@han@hosphorylation;@ndeed,@ny@modification@hat@enerates@@pecific reporter ion, tag, or neutral loss may be amenable to investigation using this technique.

\section{Acknowledgments}

This work was supported by grants awarded by the Biotechnology and Biological Sciences Research Council and the Leukaemia Research Fund.

\section{References}

1. Gu, W.; Roeder, R. G. Activation of p53 Sequence-specific DNA Binding by Acetylation of the p53 C-Terminal Domain. Cell 1997, 90, 595-606.

2. Waltzer, L.; Bienz, M. Drosophila CBP Represses the Transcription Factor TCF to Antagonize Wingless Signalling. Nature 1998, 395, 521-525.

3. Takemura, R.; Okabe, S.; Umeyama, T.; Kanai, Y.; Cowan, N. J.; Hirokawa, N. Increased Microtubule Stability and $\alpha$ Tubulin Acetylation in Cells Transfected with Microtubule-associated Proteins MAP1B, MAP2 or $\tau$. J. Cell Sci. 1992, 103, 953-964.

4. Kouzarides, T. Acetylation: A Regulatory Modification to Rival Phosphorylation? EMBO J. 2000, 19, 1176-1179.

5. Dormeyer, W.; Ott, M.; Schnolzer, M. Probing Lysine Acetylation in Proteins. Mol. Cell. Proteomics 2005, 4, 1226-1239.

6. Kim, J. Y.; Kim, K. W.; Kwon, H. J.; Lee, D. W.; Yoo, J. S. Probing Lysine Acetylation with a Modification-specific Marker Ion Using High-performance Liquid Chromatography/Electrospray-Mass Spectrometry with Collision-induced Dissociation. Anal. Chem. 2002, 74, 5443-5449.

7. Unwin, R. D.; Griffiths, J. R.; Leverantz, M. K.; Grallert, A.; Hagan, I. M.; Whetton, A. D. Multiple Reaction Monitoring to Identify Sites of Protein Phosphorylation with High Sensitivity. Mol. Cell. Proteomics 2005, 4, 1134-1144.

8. Herrmann, H.; Kreplak, L.; Aebi, U. Isolation, Characterization, and In Vitro Assembly of Intermediate Filaments. Methods Cell Biol. 2004, 78, 3-24.

9. Unwin, R. D.; Craven, R. A.; Harnden, P.; Hanrahan, S.; Totty, N.; Knowles, M.; Eardley, I.; Selby, P. J.; Banks, R .E. Proteomic Changes in Renal Cancer and Co-ordinate Demonstration of Both the Glycolytic and Mitochondrial Aspects of the Warburg Effect. Proteomics 2003, 3 , $1620-1632$.

10. Huddleston, M. J.; Bean, M. F.; Carr, S. A. Collisional Fragmentation of Glycopeptides by Electrospray Ionization LC/MS and LC/MS/MS: Methods for Selective Detection of Glycopeptides in Protein Digests. Anal. Chem. 1993, 65, 877-884.

11. Annan, R. S.; Carr, S. A. The Essential Role of Mass Spectrometry in Characterizing Protein Structure: Mapping Posttranslational Modifications. J. Protein Chem. 1997, 16, 391-402.

12. Borchers, C.; Parker, C. E.; Deterding, L. J.; Tomer, K. B. Preliminary Comparison of Precursor scans and Liquid Chromatography-Tandem Mass Spectrometry on a Hybrid Quadrupole Time-of-Flight Mass Spectrometer. J. Chromatogr. A 1999, 854, 119-130.

13. Helfand, B. T.; Chang, L.; Goldman, R. D. The Dynamic and Motile Properties of Intermediate Filaments. Annu. Rev. Cell Dev. Biol. 2003, 19, 445-467. 